\title{
Estudio de la estabilidad de cerámicas de circonato de bario en condiciones reductoras
}

\author{
P. REY, J. FRANCO, A. SOUTO, F. GUITIÁN \\ Instituto de Cerámica de Galicia. Avda Maestro Mateo S/N. Campus Sur. \\ 15782 Santiago de Compostela. España.
}

\begin{abstract}
Las cerámicas de circonato de bario $\left(\mathrm{BaZrO}_{3}\right)$ se descomponen por tratamiento térmico en condiciones reductoras en vacío. Esta descomposición se produce por volatilización selectiva del $\mathrm{BaO}$, y conduce a la aparición de partículas de circona monoclínica de elevada relación de aspecto en las superficies del material expuestas a la atmósfera reductora. La profundidad del ataque superficial y las pérdidas de peso aumentan con el tiempo y la temperatura del tratamiento. Esta inestabilidad condiciona el uso de materiales de $\mathrm{BaZrO}_{3}$ en condiciones fuertemente reductoras a altas temperaturas.
\end{abstract}

Palabras clave: circonato de bario, reducción superficial, circona monoclinica, condiciones de equilibrio.

Stability of barium zirconate ceramics under reducing conditions.

The stability of barium zirconate $\left(\mathrm{BaZrO}_{3}\right)$ components in reducing conditions at high temperatures has been studied. The experiments consisted in thermal treatments at temperatures in the range $1300^{\circ} \mathrm{C}-1500^{\circ} \mathrm{C}$, for times up to 5 hours, under vacuum (absolute pressure: $10 \mathrm{~Pa}$ ). Weight losses up to 3\% have been achieved. XRD and SEM characterization of the ceramics after the above experiments showed the presence of high aspect-ratio monoclinic zirconia particles on the surfaces of the ceramics. These results can be explained in terms of selective volatilization of $\mathrm{BaO}$, and must be considered for using barium zirconate ceramics at high temperatures under reducing environments.

Keywords: Barium zirconate, surface reduction, monoclinic zirconia, equilibrium conditions.

\section{INTRODUCCIÓN}

Las cerámicas de circonato de bario $\left(\mathrm{BaZrO}_{3}\right)$ encuentran crecientes aplicaciones en la tecnología actual, debido a sus singulares propiedades físicas. $\mathrm{El} \mathrm{BaZrO}_{3}$ funde congruentemente a una temperatura aproximada de $2600^{\circ} \mathrm{C}$, lo que lo convierte en un candidato aventajado para su uso como refractario, en el campo de las barreras térmicas, en la fabricación de composites cerámicos o en la industria aeroespacial $(1,2,3)$. Además de su alto punto de fusión, su bajo coeficiente de dilatación, baja conductividad térmica y buenas propiedades mecánicas lo convierten en un candidato idóneo para su uso en la fabricación de crisoles para tratamientos térmicos y fusiones de una variedad muy amplia de materiales. Estas propiedades, unidas a su estabilidad frente a la mayoría de ácidos y bases, y su resistencia al ataque de fundidos a altas temperaturas, convierten a los crisoles de $\mathrm{BaZrO}_{3}$ en los más adecuados para el crecimiento de cristales superconductores de $\mathrm{YBaCuO}(4,5)$. Con este fin se han fabricado tanto crisoles monolíticos (6) como recubrimientos de $\mathrm{BaZrO}_{3}$ sobre distintos sustratos $(7,8)$.

En este trabajo se estudia la estabilidad de crisoles de $\mathrm{BaZrO}_{3}$ en condiciones reductoras de baja presión parcial de $\mathrm{O}_{2}(\mathrm{~g})$ a altas temperaturas, bajo una presión absoluta de $10^{-4}$ atm (10Pa). La volatilización total o parcial de los materiales cerámicos representa un hecho de gran importancia desde un punto de vista tecnológico, y ha sido estudiado en profundidad
$(9,10)$. En ocasiones, este tipo de reacciones ha sido empleado en la obtención de recubrimientos superficiales $(11,12)$. Para la fabricación de los crisoles se ha seguido una ruta de síntesis que permite obtener polvo de $\mathrm{BaZrO}_{3}$ de tamaño submicrónico, adecuado para la sinterización de crisoles con densidades próximas a la teórica a temperaturas del orden de $1600^{\circ} \mathrm{C}$. Rutas alternativas de síntesis de polvo de $\mathrm{BaZrO}_{3}$ de tamaño submicrónico aparecen en la literatura reciente (1315). Los resultados de los tratamientos térmicos se interpretan en base a las distintas estabilidades de los componentes del material en condiciones reductoras.

\section{PROCEDIMIENTO EXPERIMENTAL}

El polvo de $\mathrm{BaZrO}_{3}$ se sintetizó por reacción entre nanopartículas de un precursor amorfo de circona $\left(\mathrm{ZrO}_{2} \cdot \mathrm{xH}_{2} \mathrm{O}\right)$ y de carbonato de bario $\left(\mathrm{BaCO}_{3}\right)$. El precursor de la circona se obtuvo por hidrólisis de $\mathrm{ZrCl}_{4}$ (Merck) y posterior alcalinización de la disolución con $\mathrm{NH}_{3(\mathrm{aq})}$. El tamaño de partícula del material así obtenido fue $100 \%<0,1 \mu \mathrm{m}$ (medido en un Sedigraph Micromeritics 5100). El carbonato de bario se obtuvo por precipitación a partir de una mezcla de disoluciones de $\mathrm{BaCl}_{2}$ (Panreac) y $\mathrm{Na}_{2} \mathrm{CO}_{3}$.(Panreac). Tras el filtrado se obtuvo un polvo de carbonato de bario $100 \%<0,1 \mu \mathrm{m}$. 
Una vez precipitadas, las suspensiones de $\mathrm{ZrO}_{2} \cdot \mathrm{xH}_{2} \mathrm{O}$ y $\mathrm{BaCO}_{3}$ se lavaron repetidas veces con agua desionizada para eliminar los cloruros residuales, filtrándose el producto entre cada lavado. A continuación se mezclaron las cantidades adecuadas de cada una de ellas para una relación molar 1:1, manteniéndose en agitación constante durante 24 horas. Tras el proceso de mezclado se introdujeron en una estufa a $110^{\circ} \mathrm{C}$ por espacio de 24 horas. El posterior tratamiento térmico de este producto durante 3 horas a $1000^{\circ} \mathrm{C}$ condujo a la cristalización del circonato de bario. En la Figura 1 se muestra el análisis por Difracción de Rayos X de este producto (DRX, modelo Siemens D5000, operando con la radiación $\mathrm{Ka}$ del $\mathrm{Cu}$ a $25 \mathrm{kV}$ y $35 \mathrm{~mA}$, con una velocidad de barrido de $2^{\circ} / \mathrm{min}$ ). En la figura 2 se muestra la curva de distribución de tamaño de partícula para el polvo de circonato de bario sintetizado, en la que se ve que el 95\% de las partículas tienen un tamaño inferior a $2 \mu \mathrm{m}$. El tamaño medio del polvo de circonato de bario (D50) es de $0,7 \mu \mathrm{m}$.

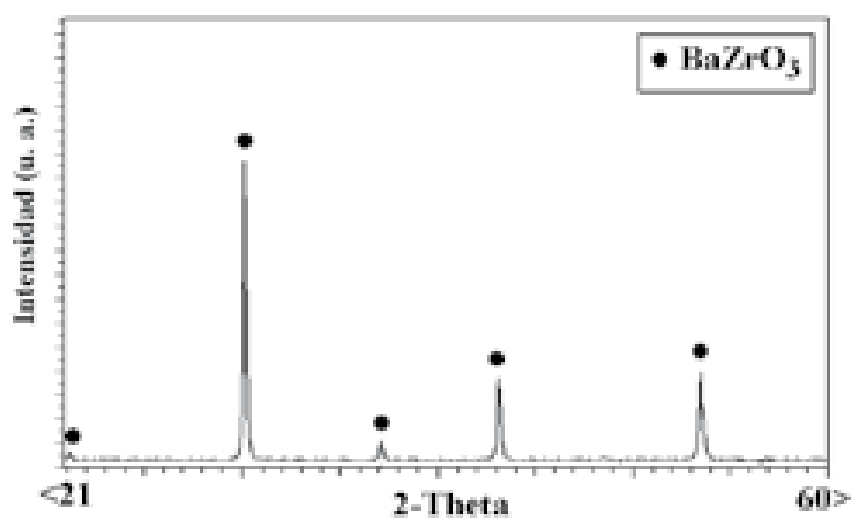

Fig. 1- Análisis por Difracción de Rayos X del polvo de $\mathrm{BaZrO}_{3}$.

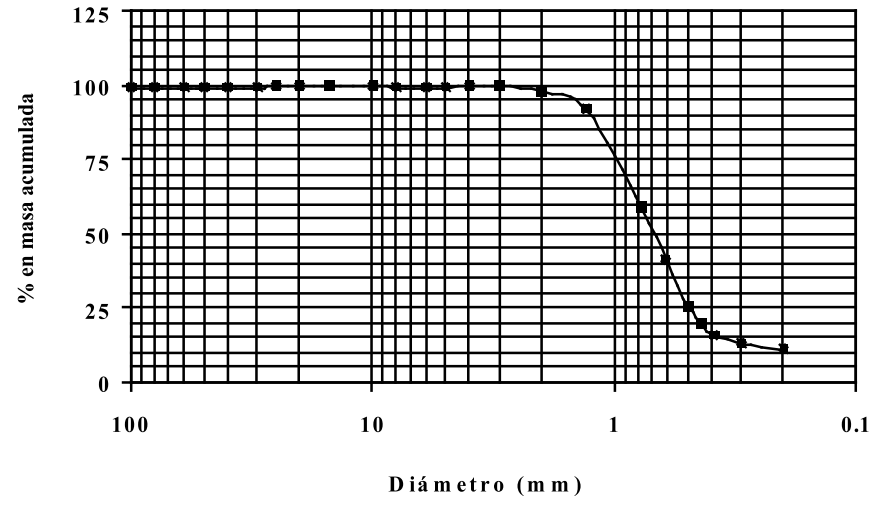

Fig. 2- Análisis granulométrico del polvo de circonato de bario sintetizado.

Los crisoles de $\mathrm{BaZrO}_{3}$ se fabricaron por colado. Para ello, se prepararon barbotinas de polvo de circonato de bario, con hexametafosfato sódico como dispersante $(2 \%)$. El contenido en sólidos de las barbotinas fue del $80 \%$ en peso. Una vez procesados y desmoldados, los crisoles se secaron al aire durante 24 horas. El secado final se realizó en una estufa a $110^{\circ} \mathrm{C}$ durante 1 hora. El tratamiento térmico de estos crisoles consistió en un calentamiento a $1600^{\circ} \mathrm{C}$ durante 12 horas. La

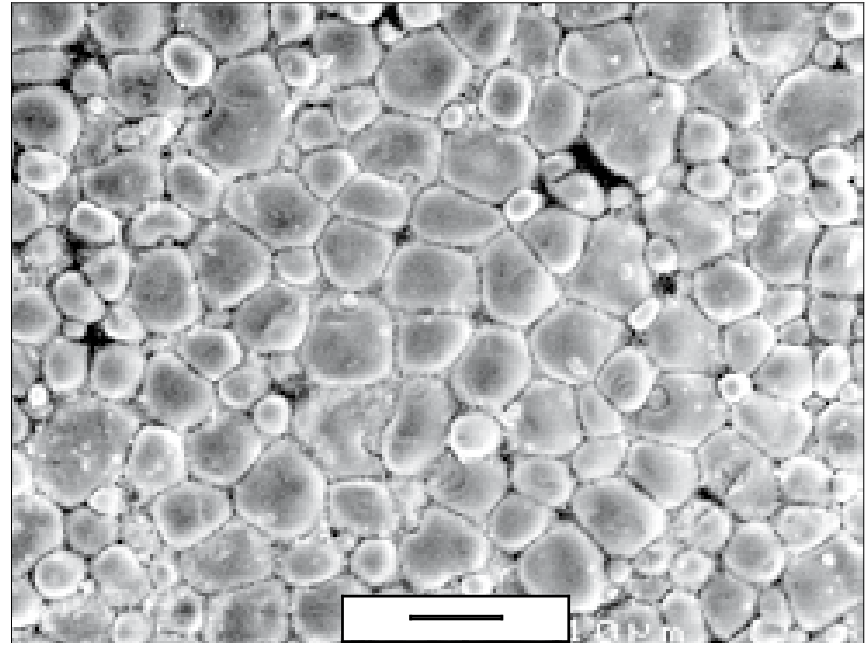

Fig. 3- Microestructura de los crisoles de $\mathrm{BaZrO}_{3}$ después de 12 horas a $1600^{\circ} \mathrm{C}$ (Barra de escala: $\left.10 \mu \mathrm{m}\right)$.

velocidad de subida y bajada de temperatura fue $10^{\circ} \mathrm{C} / \mathrm{min}$. La porosidad residual fue del $10 \%$, con un tamaño medio de poro de $0,5 \mu \mathrm{m}$ (Porosimetría de mercurio, Micromeritics Autopore II 9215). Las microestructuras se estudiaron por microscopía electrónica de barrido (SEM, Modelo JEOL JSM-6400). Las variaciones de peso asociadas a los distintos tratamientos se determinaron en una balanza analítica Mettler AE200, con una precisión de $\pm 0.1 \mathrm{mg}$.

Para evaluar su comportamiento y estabilidad, los crisoles fueron tratados térmicamente en atmósferas reductoras, en distintas condiciones de tiempo y temperatura, bajo una presión absoluta constante de $10^{-4} \mathrm{~atm}(10 \mathrm{~Pa})$. Los ensayos se realizaron en un horno de grafito Pyrox HI-16L/17. Una vez cargados los crisoles y cerrado el horno se conectó la bomba de vacío del horno, hasta alcanzar una presión interna de $10^{-4}$ atm (10 Pa). El calentamiento se inició en estas condiciones. Esta secuencia de purgado asegura una presión parcial de $\mathrm{O}_{2}(\mathrm{~g})$ inicial de aproximadamente $2 \times 10^{-5} \mathrm{~atm}(2 \mathrm{~Pa})$, que se transforma totalmente en $\mathrm{CO}(\mathrm{g})$ a altas temperaturas por reacción con los componentes de grafito del horno. La bomba de vacío se mantuvo en funcionamiento durante todo el

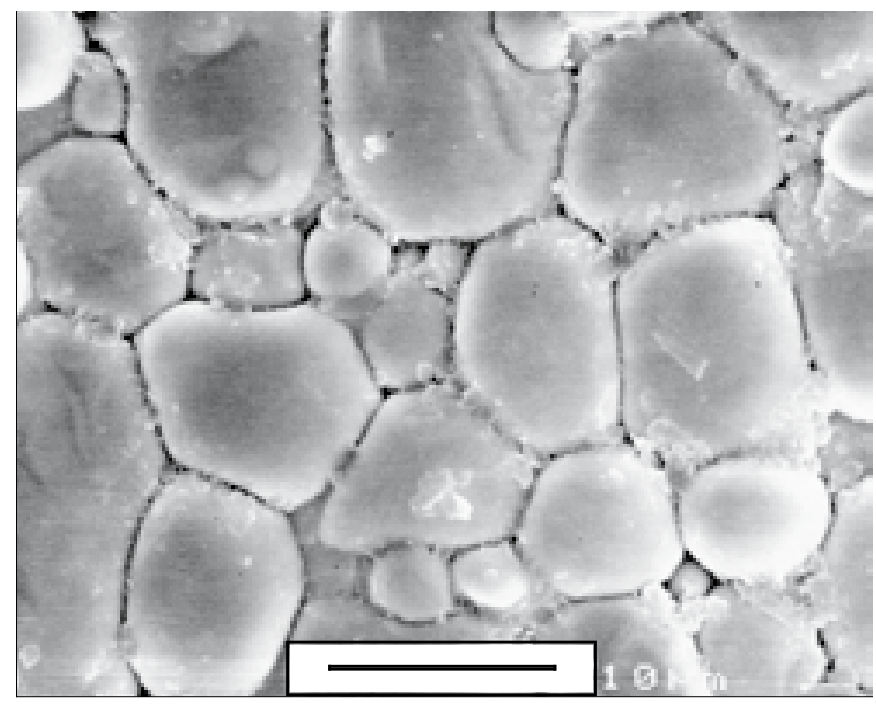

Fig. 4- Detalle de la microestructura de los crisoles de $\mathrm{BaZrO}_{3}$ después de 12 horas a $1600^{\circ} \mathrm{C}$ (Barra de escala: $\left.10 \mu \mathrm{m}\right)$. 


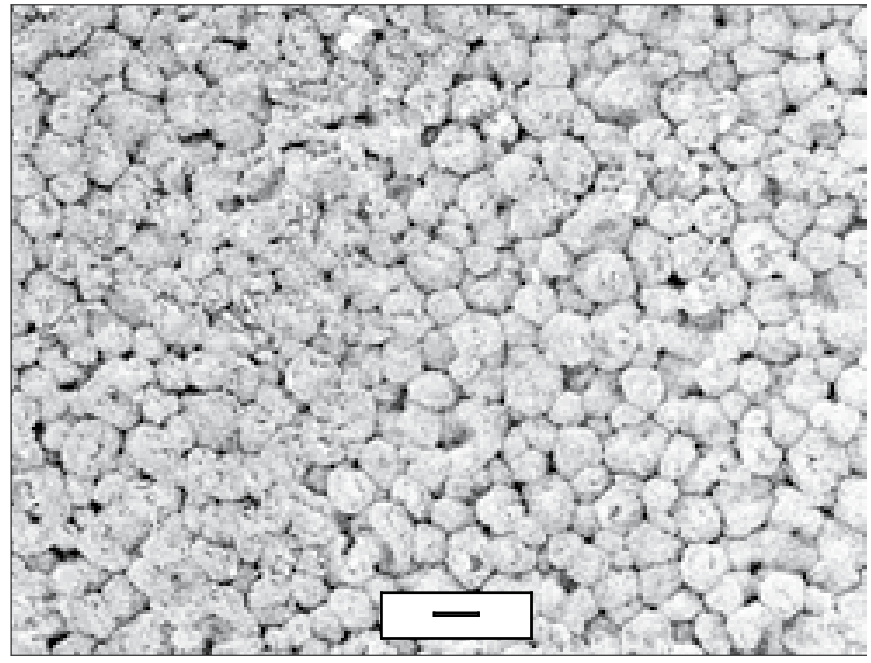

Fig. 5- Aspecto general por SEM de la superficie del $\mathrm{BaZrO}_{3}$ después de tratamientos térmicos en condiciones reductoras, bajo una presión absoluta de $10^{-4} \mathrm{~atm}(10 \mathrm{~Pa})$ (Barra de escala: $10 \mu \mathrm{m}$ ).
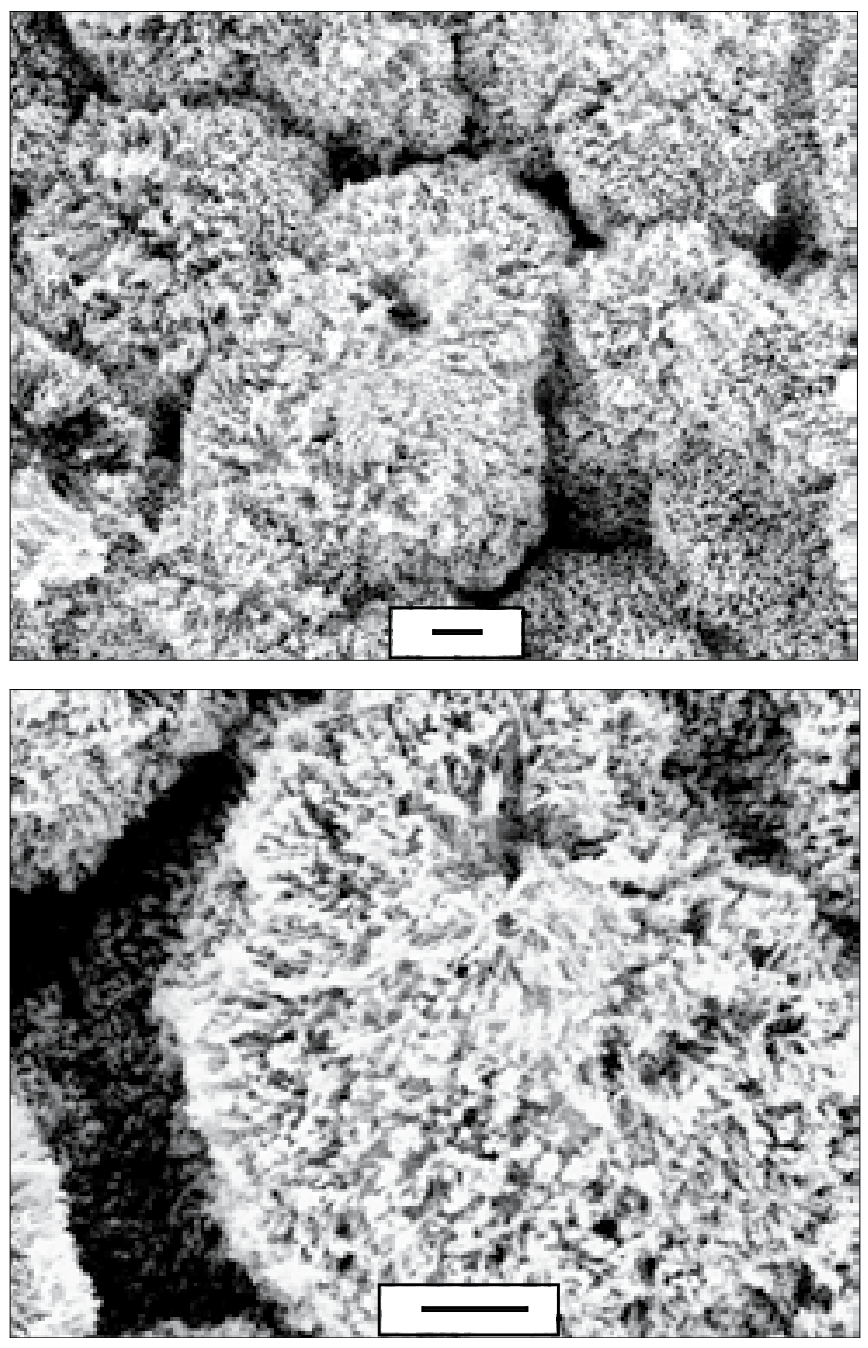

Fig. 6- Microestructuras de la superficie del $\mathrm{BaZrO}_{3}$ después de su tratamiento térmico en condiciones reductoras, bajo una presión absoluta de $10^{-4} \mathrm{~atm}(10 \mathrm{~Pa})$ (Barra de escala: $\left.1 \mu \mathrm{m}\right)$.

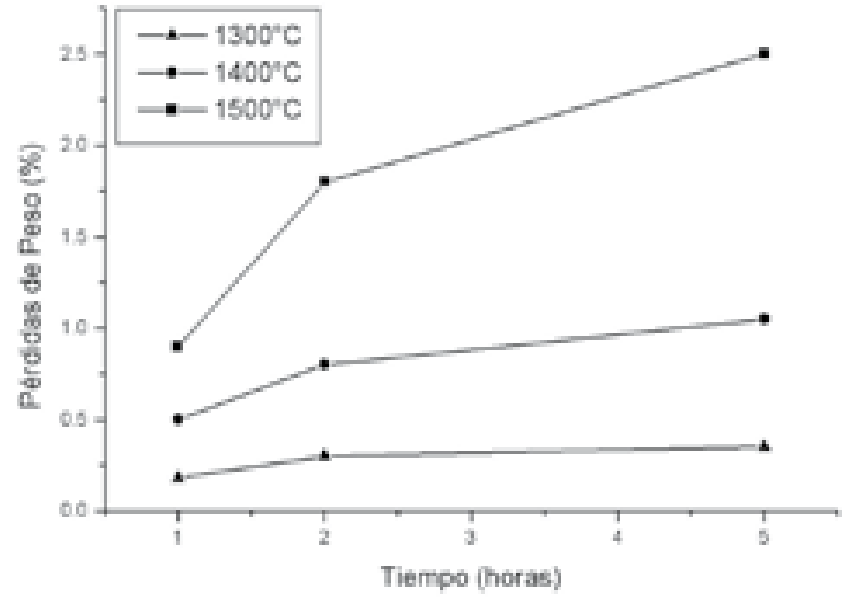

Fig. 7- Pérdidas de peso experimentadas por crisoles de $\mathrm{BaZrO}_{3}$ después de tratamientos térmicos reductores, a las temperaturas de $1300^{\circ} \mathrm{C}, 1400^{\circ} \mathrm{C}$ y $1500^{\circ} \mathrm{C}$, durante 1,2 , y 5 horas, bajo una presión absoluta de $10^{-4} \mathrm{~atm}(10 \mathrm{~Pa})$.

tratamiento, para mantener una presión absoluta constante de aproximadamente $10^{-4}$ atm (10 Pa) en el interior del horno. La velocidad de subida de temperatura es de $10^{\circ} \mathrm{C} / \mathrm{min}$, y las temperaturas de tratamiento oscilan entre $1300^{\circ} \mathrm{C}$ y $1500^{\circ} \mathrm{C}$, durante tiempos de hasta 5 horas.

\section{RESULTADOS Y DISCUSIÓN}

El análisis de los crisoles por SEM después de 12 horas a $1600^{\circ} \mathrm{C}$ muestra una microestructura constituida por partículas de circonato de bario, con granos que alcanzan las $10 \mu \mathrm{m}$ (Figura 3). La observación de esta superficie a más aumentos (Figura 4) muestra la presencia de cantidades apreciables de fases intercristalinas y porosidad residual concentrada en bordes de grano y puntos triples.

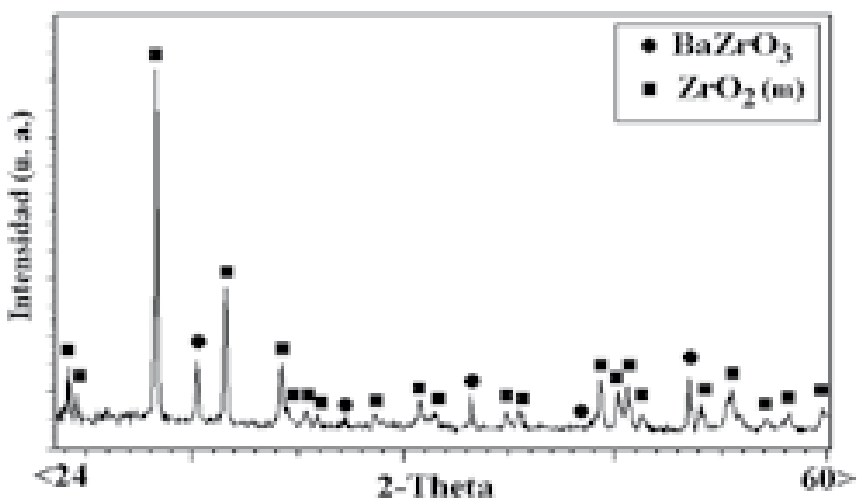

Fig. 8- Análisis por Difracción de Rayos X de la superficie de los crisoles después de un tratamiento térmico de 3 horas a $1450^{\circ} \mathrm{C}$ en condiciones reductoras, bajo una presión absoluta de $10^{-4} \mathrm{~atm}(10 \mathrm{~Pa})$.

Los experimentos realizados no han producido ningún cambio macroscópico ni morfológico en los crisoles. Donde sí se encuentran importantes alteraciones es en la microestructura de sus superficies, como puede apreciarse en la figura 5. El 
estudio comparativo de las Figura 3, 4, y 5 demuestra que la superficie del $\mathrm{BaZrO}_{3}$ expuesta a la atmósfera reductora resulta degradada, presentando un aspecto muy poroso como consecuencia de los tratamientos térmicos. La observación a más aumentos muestra que estas superficies están constituidas por microcristales de muy pequeño tamaño, de formas fibrosas y elevada relación de aspecto. El diámetro de estas partículas es de aproximadamente $100 \mathrm{~nm}$ (Figura 6). Para cuantificar la intensidad del ataque se han medido las pérdidas de peso en los distintos ensayos realizados. Los datos obtenidos demuestran que las pérdidas aumentan con el tiempo y la temperatura de tratamiento, pudiendo alcanzar porcentajes próximos al 3\% (Figura 7).

El análisis de estas superficies por DRX demuestra que el producto final del ataque es en todos los casos circona monoclínica. A modo de ejemplo, en la Figura 8 aparece el difractograma correspondiente a un ataque de 3 horas a $1450^{\circ} \mathrm{C}$. Además de los picos de circona se detectan pequeños picos de circonato de bario, pertenecientes al material inalterado situado por debajo de la circona.

Los experimentos descritos demuestran que el circonato de bario se descompone en condiciones reductoras, perdiendo selectivamente $\mathrm{BaO}$. $\mathrm{El} \mathrm{ZrO}_{2}$ permanece estable e inalterado en las superficies del crisol. Estos resultados son consecuencia de la diferente estabilidad termoquímica de los dos óxidos que forman parte del circonato de bario. A temperaturas elevadas y en atmósferas reductoras como las empleadas en este estudio, la degradación del $\mathrm{BaZrO}_{3}$ puede transcurrir por distintos mecanismos. En primer lugar, esta reacción puede producirse por descomposición directa, según las ecuaciones siguientes (reacciones 1 y 2 ):

$$
\begin{aligned}
& \mathrm{BaZrO}_{3} \rightarrow \mathrm{ZrO}_{2}+\mathrm{BaO}(\mathrm{g}) \quad K_{P}=P_{\mathrm{BaO}} \\
& \mathrm{BaZrO}_{3} \rightarrow \mathrm{ZrO}_{2}+\mathrm{Ba}(\mathrm{g})+1 / 2 \mathrm{O}_{2}(\mathrm{~g}) \quad K_{P}=P_{B a} \cdot P_{O_{2}}^{1 / 2}
\end{aligned}
$$

El segundo mecanismo posible es la reacción con el $\mathrm{CO}(\mathrm{g})$ de la atmósfera del horno. Asumiendo reacción total entre el $\mathrm{O}_{2}(\mathrm{~g})$ residual presente en el horno después de la secuencia de purgado y los componentes de grafito de éste, la presión parcial de $\mathrm{CO}(\mathrm{g})$ puede estimarse en $4 \times 10^{-5} \mathrm{~atm}(4 \mathrm{~Pa})$. La ecuación de este proceso es (reacción 3):

$$
\mathrm{BaZrO}_{3}+\mathrm{CO}(\mathrm{g}) \rightarrow \mathrm{ZrO}_{2}+\mathrm{Ba}(\mathrm{g})+\mathrm{CO}_{2}(\mathrm{~g}) K_{P}=\frac{P_{B a} \cdot P_{C O_{2}}}{P_{C O}}
$$

A modo de ejemplo, a $1500^{\circ} \mathrm{C}$, las constantes de equilibrio para las reacciones 1,2 , y 3 son, respectivamente, $1.7 \times 10$ ${ }^{4}, 2.1 \times 10^{-9}$ y $1.4 \times 10^{-7}$ (datos extraídos de Factsage ${ }^{\mathrm{TM}} 5.1$ Thermochemical Software and Database Package). Asumiendo un comportamiento ideal e igual difusividad de los gases, el equilibrio de la reacción 1 se alcanza con una presión parcial de $\mathrm{BaO}(\mathrm{g})(\mathrm{P} \mathrm{BaO})$ de $1.7 \times 10^{-4} \mathrm{~atm}(17.2 \mathrm{~Pa})$. Teniendo en cuenta el criterio de balance de masas para el cual se asume que $\mathrm{P}_{\mathrm{Ba}}=2 \mathrm{P}_{\mathrm{O}}$, la reacción 2 alcanza el equilibrio cuando la $\mathrm{P}_{\mathrm{Ba}}=1.9 \times 10^{-6}$ atm $(0.2 \mathrm{~Pa})$ y la $\mathrm{P}_{\mathrm{O}_{2}}=9.5 \times 10^{-7} \mathrm{~atm}(0.1 \mathrm{~Pa})$. Suponiendo nuevamente un comportamiento ideal de los gases, las presiones parciales de $\mathrm{Ba}(\mathrm{g})$ y $\mathrm{CO}_{2}(\mathrm{~g})$ son iguales. Teniendo esto en cuenta, y siendo la presión parcial de $\mathrm{CO}(\mathrm{g})$ de $4 \times 10^{-5}$ atm $(4 \mathrm{~Pa})$, la reacción 3 está en equilibrio cuando
$\mathrm{P}_{\mathrm{Ba}}=\mathrm{P}_{\mathrm{CO}_{2}}=2.4 \times 10^{-6} \mathrm{~atm}(0.2 \mathrm{~Pa})$. Estas presiones parciales, especialmente las producidas por la reacción 1, determinan que la descomposición del $\mathrm{BaZrO}_{3}$ sea un proceso a tener en cuenta al valorar sus posibles aplicaciones.

$\mathrm{Al}$ igual que el $\mathrm{BaZrO}_{3}$, las partículas de $\mathrm{ZrO}_{2}$ formadas en las reacciones anteriores están expuestas directamente a la atmósfera reductora y pueden reaccionar con ella. Las posibles reacciones de reducción de la circona son las siguientes:

$$
\begin{aligned}
& \mathrm{ZrO}_{2} \rightarrow \mathrm{ZrO}(\mathrm{g})+1 / 2 \mathrm{O}_{2}(\mathrm{~g}) \quad K_{P}=P_{\mathrm{ZrO}} \cdot P_{\mathrm{O}_{2}}^{1 / 2} \\
& \mathrm{ZrO}_{2}+\mathrm{CO}(\mathrm{g}) \rightarrow \mathrm{ZrO}(\mathrm{g})+\mathrm{CO}_{2}(\mathrm{~g}) K_{P}=\frac{P_{\mathrm{ZrO}} \cdot P_{\mathrm{CO}_{2}}}{P_{\mathrm{CO}}}
\end{aligned}
$$

Asumiendo un comportamiento ideal de los gases y teniendo en cuenta el criterio de balance de masas, la reacción 4 alcanza el equilibrio a $1500^{\circ} \mathrm{C}$ para una presión de $\mathrm{O}_{2}$ de $2 \times 10^{-10} \mathrm{~atm}\left(2 \times 10^{-5} \mathrm{~Pa}\right)$. En estas condiciones, $\mathrm{P} \mathrm{ZrO}=4 \times 10^{-10}$ atm $\left(4 \times 10^{-5} \mathrm{~Pa}\right)$. Para una $\mathrm{P}_{\mathrm{CO}}=4 \times 10^{-5}$ atm $(4 \mathrm{~Pa})$, la reacción 5 alcanza el equilibrio a $1500^{\circ} \mathrm{C}$ cuando $\mathrm{P}_{\mathrm{ZrO}}=\mathrm{PCO}_{2}=5,5 \times 10^{-9}$ atm $\left(5.6 \times 10^{-4} \mathrm{~Pa}\right)$.

De acuerdo con estos cálculos, las reacciones 4 y 5 alcanzan sus equilibrios con presiones parciales varios órdenes de magnitud inferiores a las producidas por el $\mathrm{BaZrO}_{3}$. Estas bajas presiones parciales de equilibrio determinan que los efectos de estas reacciones sean inapreciables a efectos prácticos. Por tanto, una vez formadas las partículas superficiales de $\mathrm{ZrO}_{2}$ se mantienen inalteradas durante todo el tratamiento térmico.

\section{CONCLUSIONES}

Los resultados que se presentan en este trabajo demuestran que el circonato de bario se descompone a altas temperaturas en vacío, perdiendo selectivamente $\mathrm{BaO}$. El resultado de esta volatilización selectiva es la formación de pequeñas partículas de $\mathrm{ZrO}_{2}$ pura, la cual transforma a la fase monoclínica durante el enfriamiento. La intensidad del ataque y las pérdidas de peso aumentan con el tiempo y la temperatura del tratamiento. Se ha comprobado que de entre todos los posibles mecanismos de reacción, la volatilización directa como $\mathrm{BaO}(\mathrm{g})$ es en el que se alcanzan las mayores presiones parciales de equilibrio. La inestabilidad del circonato de bario en atmósferas reductoras de baja presión absoluta a altas temperaturas debe ser tenida en cuenta para potenciales usos de crisoles y demás componentes de este material en dichas condiciones.

\section{BIBLIOGRAFÍA}

1. A. Azad, A. Subramaniam, T. W. Dung, On the Development of High Density Barium Metazirconate (BaZrO ${ }_{3}$ ) Ceramics, J. Alloys Compd. 334, 118-130 (2002).

2. G. M. Gladysz, M. Schmücker, K. K. Chawla, H. Schneider, D. L. Joslin, M. K. Ferber, Characterization of the Reaction Products that Develop in the Processing of $\mathrm{Al}_{2} \mathrm{O}_{3} / \mathrm{BaZrO}_{3}$ Laminated Composites, Mater Charact. 40, 209-214 (1998).

3. R. Vassen, X. Cao, F. Tietz, D. Basu, D. Stöver, Zirconates as New Materials for Thermal Barrier Coatings, J. Am. Ceram. Soc. 83, 8, 2023-28 (2000).

4. A. Erb, E. Walter, R. Flükiger, $\mathrm{BaZrO}_{3}$ : the Solution for the Crucible Corrosion Problem During the Single Crystal Growth of High-Tc Superconductors $\mathrm{REBa}_{2} \mathrm{Cu}_{3} \mathrm{O}_{7-8} ; \mathrm{RE}=\mathrm{Y}$, Pr, Physica C, 245, 245-251 (1995).

5. R. Liang, D. A. Bonn, W. N. Hardy, Growth of High Quality Single Crystals Using $\mathrm{BaZrO}_{3}$ Crucibles, Physica C, 304, 105-111 (1998). 
6. N. Alford, A. Templeton, S. J. Penn, A simple Process for manufacturing Barium Zirconate Crucibles, Supercond. Sci. Technol. 11, 703 (1998).

7. J. C. Schmidt, A. Tigges, G. J. Schmitz, Synthesis of Polycrystalline $\mathrm{BaZrO}_{3}$ Coatings, Mater. Sci. Eng. B, 53, 115-118 (1998).

8. B. Robertz, A. Rulmont, B. Gilbert, R. Cloots, M. Ausloos, N. Leroy, Inhibition of the Macrocrack Formation in Zirconia Substrates by Barium Zirconate Formation, Mater. Lett. 41, 273-277 (1999).

9. V. L. K. Lou, T. E. Mitchell and A. H. Heuer, Review-Graphical Displays of the thermodynamics of high temperature gas-solid reactions and their application to oxidation of metals and evaporation of oxides, J. Am. Ceram. Soc. 68, 2, 49-58 (1985)

10. E. J. Opila, Oxidation and Volatilization of Silica Formers in Water Vapor, J. Am. Ceram. Soc. 86, 8, 1238-1249 (2003).
11. A. Souto and F. Guitián, Novel Method for Obtaining Corundum Layers of High Surface Area on Ceramic Supports for High-Temperature Catalysis, J. Am. Ceram. Soc. 85, 7, 1823-26 (2002).

12. P. Rey, A. Souto, J. Franco, F. Guitian, Reducción Superficial de Circón en Atmósferas de Baja $\mathrm{PO}_{2}$ a Altas Temperaturas, Bol. Soc. Esp. Ceram. V. 45, 1, 33-37 (2006)

13. A. Azad, S. Subramaniam, Synthesis of $\mathrm{BaZrO}_{3}$ by a Solid-State Reaction Technique Using Nitrate Precursors, Mater. Res. Bull. 37, 85-97 (2002).

14. A. Sin, B. El Montaser, P. Odier, F. Weiss, Synthesis and Sintering of Large Batches of Barium Zirconate Nanopowders, J. Am. Ceram. Soc. 85, 8, 192832 (2002).

15. A. Ubaldini, V. Buscaglia, C. Uliana, G. Costa, M. Ferretti, Kinetics and Mechanism of Formation of Barium Zirconate from Barium Carbonate and Zirconia Powders, J. Am. Ceram. Soc. 86, 1, 19-25 (2003). 